\title{
Complete Spectral Assignment of Acrylonitrile/Vinylidene Chloride Copolymers by One and Two Dimensional NMR Spectroscopy
}

\author{
Ajaib Singh Brar, ${ }^{\dagger}$ Kaushik Dutta, and Deepak PandeY \\ Department of Chemistry, Indian Institute of Technology, \\ Hauz Khas, New Delhi 110 016, India
}

(Received November 11, 1998)

\begin{abstract}
KEY WORDS Acrylonitrile/Vinylidene Chloride Copolymers / 2D NMR Spectroscopy / Spectral Assignment /
\end{abstract}

Over the last decade both one and two dimensional NMR spectroscopy have become an important technique for investigations of the structure of polymers ${ }^{1-3}$ and copolymers. $^{4-6}$ Acrylonitrile/vinylidene chloride (A/V) copolymers has been extensively studied as it is an industrially important polymer. It has potential industrial application because of its excellent fiber formability together with high non-flammability. Many workers have reported the microstructure ${ }^{7-10}$ and reactivity ratios ${ }^{7-15}$

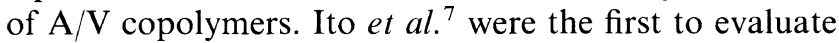
the sequence distribution of $\mathrm{A} / \mathrm{V}$ copolymers by NMR spectroscopy. Bailey et al. $^{8}$ assigned the methylene and the quaternary carbon resonance signals to dyad, triad and pentad sequences. They were not able to assign the methine and the nitrile carbon resonance signals. These signals were assigned to pentad sequences by Konig et $a l .{ }^{9}$ and Yamazaki et al. ${ }^{10}$ However, the broad and overlapping ${ }^{1} \mathrm{H}$ NMR spectra of $\mathrm{A} / \mathrm{V}$ copolymers has not been assigned by any of these workers.

In this paper we report the complete and unambiguous signal assignment of broad and overlapping ${ }^{1} \mathrm{H}$ NMR spectra of $\mathrm{A} / \mathrm{V}$ copolymers. The methine and the methylene proton signals were assigned to triad, pentad, heptad and tetrad compositional sequences, respectively, with the help of 2D NMR techniques, like DQFCOSY, TOCSY, and inverse-HETCOR. The various configurational arrangement within the tetrad or pentad sequences were also assigned.

\section{EXPERIMENTAL}

$\mathrm{A} / \mathrm{V}$ were distilled under reduced pressure and was stored below $5^{\circ} \mathrm{C}$. The $\mathrm{A} / \mathrm{V}$ chloride copolymers of different monomer composition were prepared by photo polymerization using uranyl nitrate as photo sensitizer in a sealed tube. The percent conversion was kept below $10 \%$ by precipitating the copolymers in methanol. The copolymers were purified by $N, N$-dimethylformamide (DMF)/methanol system. The copolymer composition was calculated from the percent nitrogen of the copolymers and quantitative ${ }^{13} \mathrm{C}$ NMR spectroscopy.

NMR experiments were performed in dimethyl sulfoxide (DMSO)- $d_{6}$ on a Bruker $300 \mathrm{MHz}$ spectrometer at frequencies of $300.13 \mathrm{MHz}$ and $75.5 \mathrm{MHz}$ for ${ }^{1} \mathrm{H}$ and ${ }^{13} \mathrm{C}\left\{{ }^{1} \mathrm{H}\right\}$ NMR spectra, respectively. The ${ }^{13} \mathrm{C}\left\{{ }^{1} \mathrm{H}\right\} \mathrm{NMR}$

† To whom all correspondence should be addressed. experiments were recorded with $32 \mathrm{~K}$ data points for 6000 scans with 2 s relaxation delay. The quantitative ${ }^{13} \mathrm{C}\left\{{ }^{1} \mathrm{H}\right\}$ NMR experiments were carried out using the inversegated decoupling pulse program with $12 \mathrm{~s}$ as delay time. The $90^{\circ}$ pulse for the ${ }^{1} \mathrm{H}$ and the ${ }^{13} \mathrm{C}$ nuclei used was $7.6 \mu \mathrm{s}$ and $5.5 \mu \mathrm{s}$, respectively. Two dimensional proton detected ${ }^{1} \mathrm{H}-{ }^{13} \mathrm{C}$ heteronuclear chemical shift correlation spectra were obtained using the standard pulse sequence. A total of 32 scans were accumulated with a relaxation delay of $2 \mathrm{~s}$ for each of the $512 \mathrm{t}_{1}$ experiments. The DQFCOSY spectrum was obtained with 32 scans for each $t_{1}$ value. A total of 512 spectra each containing the $1 \mathrm{~K}$ data points were accumulated. Two dimensional homonuclear total correlation spectroscopy (TOCSY) spectra were recorded with $4 \mathrm{~ms}$ mixing times. A total of 32 scans were accumulated for each of the $512 t_{1}$ experiments with a relaxation delay of $2 \mathrm{~s}$.

\section{RESULTS AND DISCUSSION}

The composition of $\mathrm{A} / \mathrm{V}$ copolymers were determined from the quantitative ${ }^{13} \mathrm{C}$ NMR spectroscopy and percent nitrogen content in the copolymers. The ${ }^{13} \mathrm{C}\left\{{ }^{1} \mathrm{H}\right\}$ NMR spectra of $\mathrm{A} / \mathrm{V}$ copolymer has been previously assigned to various compositional (dyad, triad, tetrad, and pentad) sequences. ${ }^{8,10}$ Once the carbon spectra have been assigned completely, then the corresponding proton spectrum can be assigned with the help of $2 \mathrm{D}$ experiments.

Bailey et al. ${ }^{8}$ and Yamazaki et al. ${ }^{10}$ have assigned the methylene carbon signals to dyad/tetrad compositional sequences. From the inverse-HETCOR spectrum, the methylene protons signals are divided into three regions: $\delta 1.9-2.5,2.5-3.4$, and $3.5-4.0 \mathrm{ppm}$ which are assigned to AA, AV(VA), and VV dyads. The central methylene protons in the AA centered tetrads (AAAA, AAAV, and VAAV) show stereochemical sensitivity, i.e., the central AA dyad of these tetrad can have meso and racemic configurations, which is reflected in the inverseHETCOR spectrum (Figure 1). The methylene protons in the AmA centered tetrads will be non equivalent and will show two cross peaks in the proton axis. Thus, the cross peaks at $\delta 34.67 / 2.35$ and $34.67 / 2.22 \mathrm{ppm}$ are assigned to the VAmAV tetrad. The cross peaks at $\delta 33.67 / 2.33$ and $33.67 / 2.11 \mathrm{ppm}$ are assigned to AAmAV tetrad. Similarly, for AAmAA tetrad the cross peaks due to the non equivalent protons are at $\delta 32.57 / 2.14$ and 


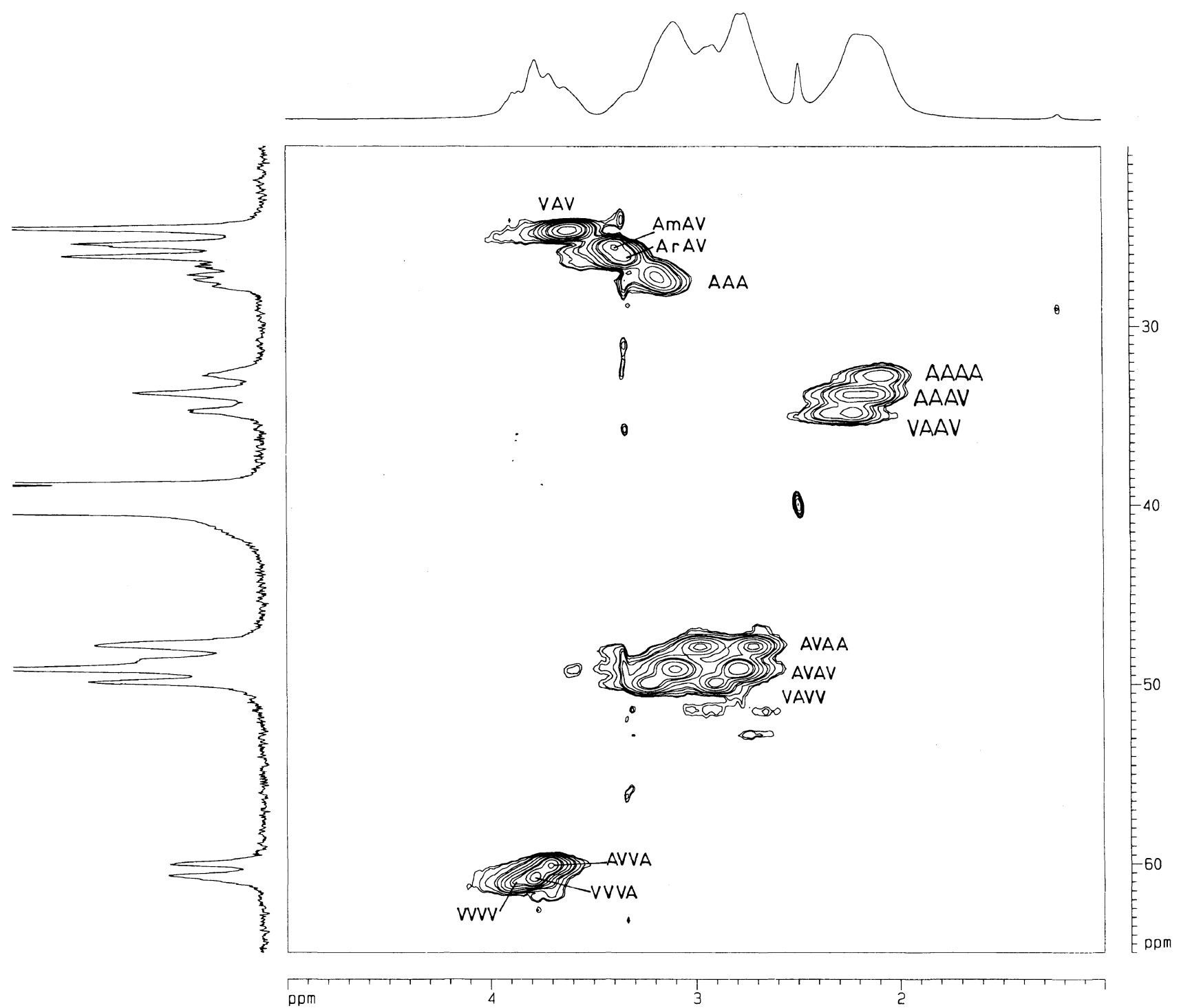

Figure 1. The inverse-HETCOR spectrum showing the methine and methylene region of acrylonitrile/vinylidene chloride (A/V) copolymer $\left(F_{\mathrm{A}}=0.57\right)$ in DMSO-d $d_{6}$.

$32.57 / 2.05 \mathrm{ppm}$. The corresponding racemic configuration tetrads are seen within these cross peaks. The geminal coupling between the non equivalent protons in these tetrads are seen in the DQFCOSY spectrum. Figure 2 shows the DQFCOSY spectrum of $\mathrm{A} / \mathrm{V}$ copolymer $\left(F_{\mathrm{A}}=0.66\right)$ in DMSO- $d_{6}$. The cross peaks at $\delta 2.35 / 2.22$, $2.23 / 2.11$, and $2.14 / 2.05 \mathrm{ppm}$ are assigned to the geminal coupling between the non equivalent proton due to meso configuration in VAmAV, VAmAA, and AAmAA tetrads, respectively.

The VA centered tetrads, AVAA ( $\delta 47.84 \mathrm{ppm})$, $\operatorname{AVAV}+\operatorname{VVAA}(\delta 49.14 \mathrm{ppm})$, and $\operatorname{VVAV}(\delta 49.87 \mathrm{ppm})$ will not show stereochemical sensitivity. The methylene protons of central VA dyad in these tetrads are non equivalent due to the non symmetrical structures of these tetrads and will show two cross peaks in the proton axis (Figure 1). The cross peaks at $\delta 47.84 / 2.97$ (47.84/2.69), $49.14 / 3.08(49.14 / 2.79)$ and $49.87 / 3.23(49.87 / 2.91) \mathrm{ppm}$ are therefore assigned to non equivalent proton in AVAA, AVAV + VVAA, and VAVV tetrads, respectively. The geminal coupling between these non equivalent protons of the $\mathrm{AV}$ centered tetrads are assigned at $\delta$ 2.97/2.69, 3.08/2.79, and 3.23/2.91 ppm (Figure 2).
Whereas the central methylene protons in the VV centered tetrads, AVVA ( $\delta 60.11 \mathrm{ppm})$, VVVA (AVVV) $(\delta 60.93 \mathrm{ppm})$, and VVVV $(\delta 61.16 \mathrm{ppm})$ are equivalent and will show one cross peaks in the inverse-HETCOR spectrum (Figure 1). The methylene protons signals at $\delta$ $3.70,3.78$, and $3.88 \mathrm{ppm}$ are assigned to AVVA, VVVA (AVVV), and VVVV tetrads, respectively.

The methine carbon signals show both stereochemical (triad) and compositional (pentad) sensitivity. ${ }^{10}$ Once the methine carbon resonance signals are completely assigned, the corresponding proton signals were assigned with the help of inverse-HETCOR spectra. The methine proton signals show both compositional and configurational sequences sensitivity. The signals around $\delta 3.70-$ $3.50, \delta 3.50-3.25$, and $\delta 3.25-3.00 \mathrm{ppm}$ are assigned to VAV, VAA (AAV), and AAA triad fractions, respectively. Various higher order compositional and configurational sequences within these triads sequences can be assigned with the help of low mixing time TOCSY spectra. In the DQFCOSY spectrum, all the crosspeaks are not seen clearly due to the cancellation of positive and negative signals. These crosspeaks can be clearly seen in the short mixing time $(4 \mathrm{~ms})$ TOCSY spectrum. At 


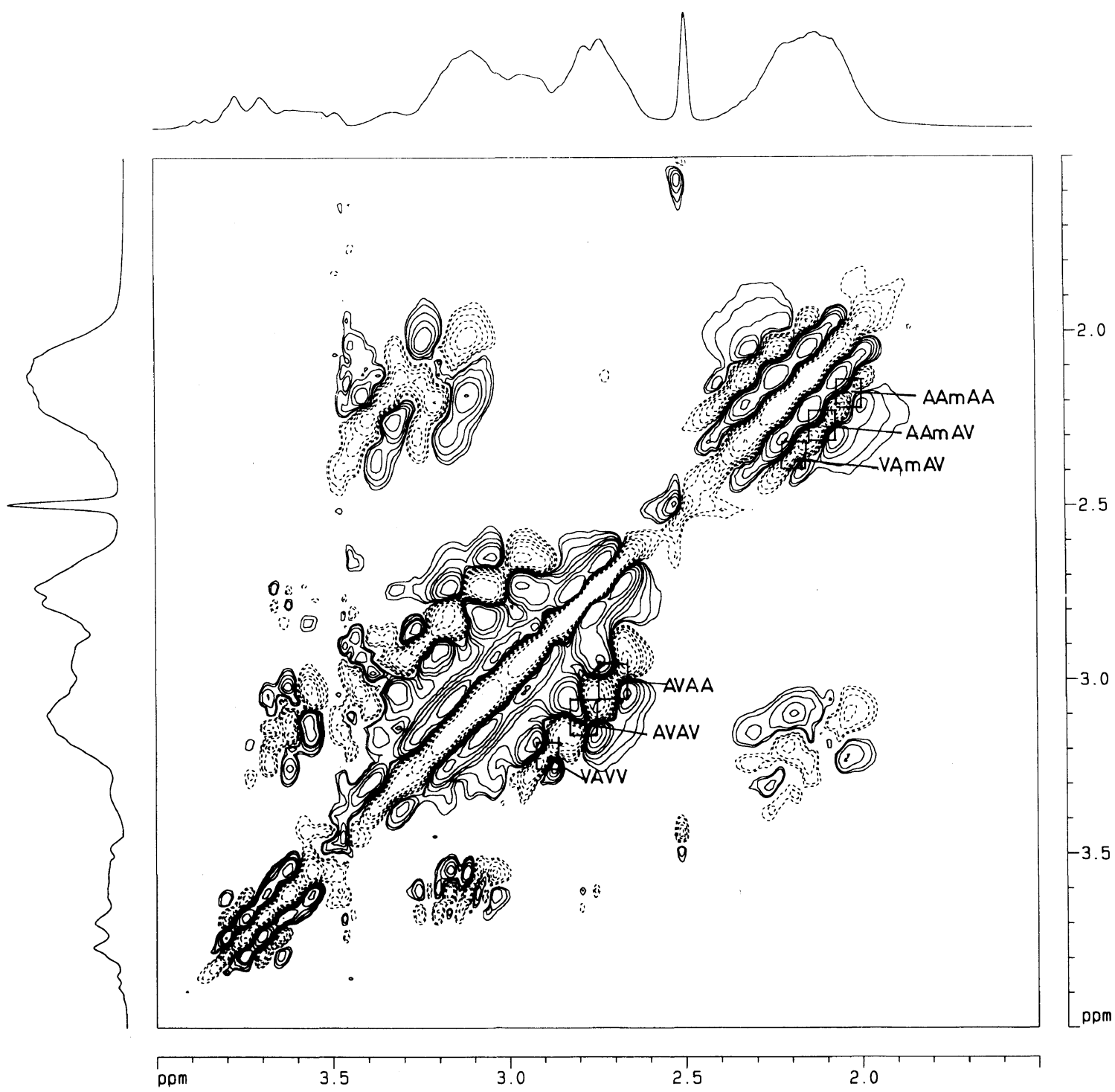

Figure 2. The 2D-DQFCOSY spectrum of $\mathrm{A} / \mathrm{V}$ copolymer $\left(F_{\mathrm{A}}=0.66\right)$ in DMSO- $d_{6}$.

shorter mixing time one can see cross peaks between the resonances of directly coupled protons (AM spin type) whereas at longer mixing time one can see cross peaks between the resonances of protons from relayed coupling (AMX spin type) through magnetization transfer.

Figures $3 \mathrm{a}$ and $3 \mathrm{~b}$ show the TOCSY spectra of $\mathrm{A} / \mathrm{V}$ copolymer of two different copolymer composition. The central methine protons of AAA and VAV triad fractions show three bond coupling to the methylene protons of AA and AV dyad regions, respectively, whereas the methine proton of AAV triad fraction shows coupling to methylene protons of both $\mathrm{AA}$ and $\mathrm{AV}$ dyad regions. From one to one correlation between the methine carbon and the corresponding proton, the methine proton signals of the AAA triad fraction are assigned around $\delta 3.12$ (ArArA), 3.15 (AmArA), and 3.18 (AmAmA) ppm. These methine protons show three bond coupling to the methylene protons, therefore, the cross peaks at $\delta 3.18 /$ 2.21 (Cross Peak 1), 3.16/2.17 (2), 3.13/2.11 (3), 3.12/2.03 (4), and 3.09/2.01 (5) ppm are assigned to AmAmA, AAmArAV, AAmArAA, AArArAV + VArArAA, and AArArAA triad sequences respectively. The central methine proton in the AAV triad shows three bond coupling with two types of methylene protons (A and V type). The cross peaks at $\delta 3.32 / 2.13$ (6) and 3.33/2.19 (7) ppm are due to the three bond coupling between the methine proton of ArAV triad fraction and the methylene protons of AA dyad. These cross peaks are assigned to AArAvA and AArAVV + VArAVA pentad sequences respectively. The three bond coupling between the methine proton of the ArAV triad fraction and the methylene protons of AV dyad are at $\delta 3.32 / 3.01$ (8), 3.33/2.97 (9), and 3.34/2.93 (10) ppm, which are assigned to AArAVA, VArAVA and AArAVV pentad sequences. Similarly, the central methine proton of AmAV triad fraction shows two type of couplings with the methylene protons, between, AmA and AV dyads. The cross peak at $3.37 / 2.28(3.37 / 3.08)(11) \mathrm{ppm}$ is assigned to AAmAVV + AAmAVA pentad sequences. Earlier it was shown that the methylene protons in the VA dyad are non equivalent and show geminal couplings. The cross peaks at $\delta 3.33 / 2.97$ (9) and 3.33/2.67 (12) ppm are due to the non equivalent methylene protons in VA dyad, which show geminal coupling at $\delta 2.97 / 2.69$ (13) ppm.

In the VAV triad fraction the central methine proton is between the two $\mathrm{V}$-unit, thus will not show any kind of configurational sensitivity. All the cross peaks in this region are therefore due to compositional sequences. The 

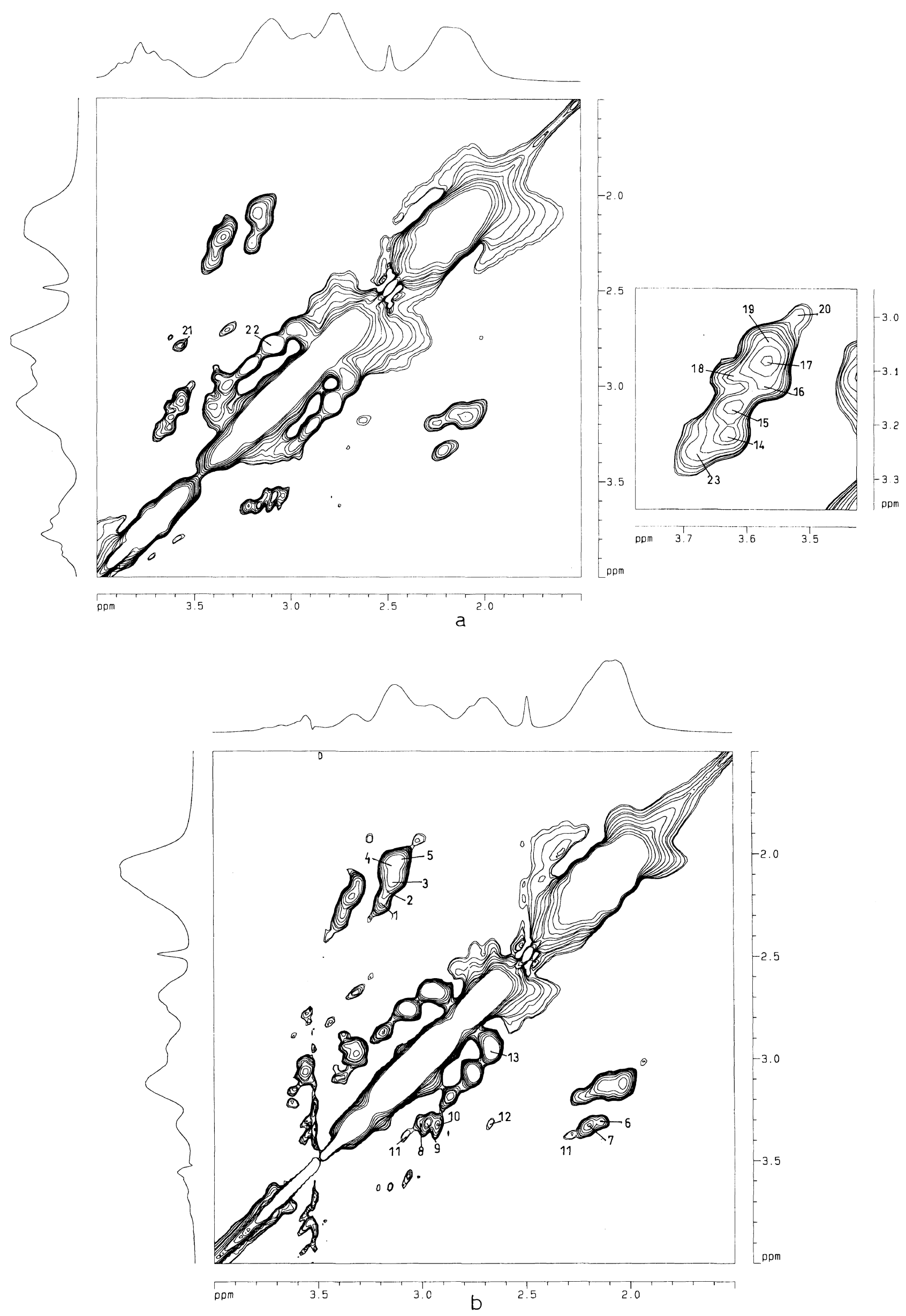

Figure 3. The $4 \mathrm{~ms} 2 \mathrm{D}-\mathrm{TOCSY}$ spectrum of the acrylonitrile/vinylidene chloride (A/V) copolymer a) $F_{\mathrm{A}}=0.57$ and b) $F_{\mathrm{A}}=0.77$ in DMSO- $d_{6}$. Polym. J., Vol. 31, No. 4, 1999 
VAV triad region can be divided into three regions: $\delta$ $3.70-3.65,3.65-3.55$, and $3.64-3.50 \mathrm{ppm}$ which are assigned to VVAVV, VVAVA + AVAVV, and AVAVA pentad sequences. The further cross peaks in VVAVA and AVAVA pentad regions are assigned to heptad sequences. The cross peaks at 3.63/3.21 (14), 3.62/3.16 (15), 3.58/3.12 (16), and 3.57/3.07 (17) are assigned to four VVAVA centered VVVAVAV, VVVAVAA, AVVAVA, and AVVAVAA heptad sequences. Similarly the AVAVA centered heptad sequences are assigned at $\delta$ 3.61/3.10 (18) (VAVAVAV), 3.57/3.03 (19) (VAVAVAA), and 3.52/2.99 (20) (AAVAVAA) ppm. The methylene protons of VA dyad are non equivalent and will show two cross peaks. Thus the cross peak at $\delta$ $3.57 / 2.79(21) \mathrm{ppm}$ is due to non equivalent proton and the corresponding geminal couplings are at 3.07/2.79 (22) ppm. The cross peak at $\delta 3.68 / 3.25$ (23) ppm is assigned to VVAVV pentad sequence, which do not show higher order compositional sequences.

\section{CONCLUSIONS}

Higher order compositional and configurational sequence assignments of the broad and overlapping proton spectrum of $\mathrm{A} / \mathrm{V}$ copolymer was done unambiguously with the help of 2D NMR experiments such as inverse-HETCOR, TOCSY.
Acknowledgments. The authors wish to thank All India Council of Technical Education, India and Department of Chemistry, Indian Institute of Technology, Delhi for providing the necessary funds and the NMR facility to carry out this work.

\section{REFERENCES}

1. M. Suchoparek and J. Spevacek, Macromolecules, 26, 102 (1993)

2. L. Dong, D. J. T. Hill, J. H. O'Donnell, and A. K. Wittaker, Macromolecules, 27, 1830 (1994).

3. C. Hijangos and D. Lopez, Macromolecules, 28, 1364 (1995).

4. A. S. Brar, K. Dutta, and G. S. Kapur, Macromolecules, 28, 8735 (1995).

5. A. S. Brar and M. Malhotra, Macromolecules, 29, 7040 (1996).

6. A. S. Brar and K. Dutta, Macromolecules, 31, 4695 (1998).

7. K. Ito, S. Kondo, Y. Yamashita, and H. Ishii, Kogyo Kagaku Zasshi, 73, 148 (1970).

8. D. B. Bailey and P. M. Henrichs, J. Polym. Sci., 16, 3185 (1978).

9. J. Konig and D. Wendish, Angew. Makromol. Chem., 98, 255 (1981).

10. H. Yamazaki, K. Okajima, and K. Kamide, Polym. J., 20, 1143 (1988).

11. A. Guyot, M. Dumont, Ch. Graillat, J. Guillot, and C. Pichot, J. Macromol. Chem., 9, 483 (1975).

12. F. Lewis, F. Mayot, and W. Hulse, J. Am. Chem. Soc., 67, 1701 (1945).

13. R. B. Parker, Jr. and B. V. Mokler, J. Polym. Sci. B, 2, 19 (1964).

14. T. Doi, M. Matsuki, and A. Sugiyama, Kogyo Kagaku Zasshi, 70, 1792 (1967)

15. L. Marker, O. J. Sweeting, and J. G. Wepsie, J. Polym. Sci., 57, 855 (1962). 